\title{
A Plan for Cities of Growth
}

\author{
Xinhua Jiang \\ North China Electric Power University Baoding, Baoding 071000, China \\ 1747634287@qq.com@163.com
}

\begin{abstract}
Keywords: Smart growth, AHP, Fuzzy Synthetic Evaluation, Multi-objective programming
Abstract. This paper proposes reasonable metric to measure the success of smart growth of a city and obtain the wise plan for cities. The whole work is carried out by means of comprehensive analysis under some hypothesized settings.

We set up five primary indicators: economic, society, environment, geographical conditions and demographics, each of which is subdivided into several secondary indicators. Analytic Hierarchy Process (AHP) Model is established to determine the weight of each indicator, which is adequately filled in the three-hierarchy structure. To overcome weakness of excess subjective factors in AHP, Fuzzy Synthetic Evaluation model is proposed to classify our indicators using data of 12 cities as samples. We divide scores into five levels in descending order: A, B, C, D and E. Combining the two models, the data is normalized, the eigenvalues and eigenvectors of judging matrix are calculated and the consistency test is conducted, which indicates robustness of our indicators classification.
\end{abstract}

\section{Introduction}

Many communities are implementing smart growth initiatives in an effort to consider long range, sustainable planning goals. Smart growth is a way to build cities, towns, and neighborhoods that are economically prosperous, socially equitable, and environmentally sustainable [1]. This task is more important than ever because the world is rapidly urbanizing. It is projected that by 2050, 66 percent of the world's population will be urban-this will result in a projected 2.5 billion people being added to the urban population [2]. Consequently, urban planning has become increasingly important and necessary to ensure that people have access to equitable and sustainable homes, resources and jobs.

These broad principles must be tailored to a community's unique needs to be effective. Thus, any measure of success must incorporate the demographics, growth needs, and geographical conditions of a city as well as the goal to adhere to the three E's.

\section{Specify evaluation norms}

In this section, for the purpose of achieving a reasonable, powerful and evident construction of the metrics system, we construct a metric incorporating the demographics, growth needs, and geographical conditions of a city as well as the goal to adhere to the three E's. It measures the ability to determine Smart growth for a country.

Building any metrics system must follow certain principles. As for the evaluation standard for Smart growth, there are mainly three aspects that count: prosperous economic, equitable society, and sustainable environment. Similarly our metric could be evaluated from 5 aspects of criteria and 26 factors of alternatives. 


\subsection{The three-hierarchy structure}

With the help of the current literatures and researching those data we searched on the internet, we develop AHP model considering five indicators in criteria level, there are economic, society, environment, geographical conditions and demographics. The three-hierarchy structure is shown in Figure 1.
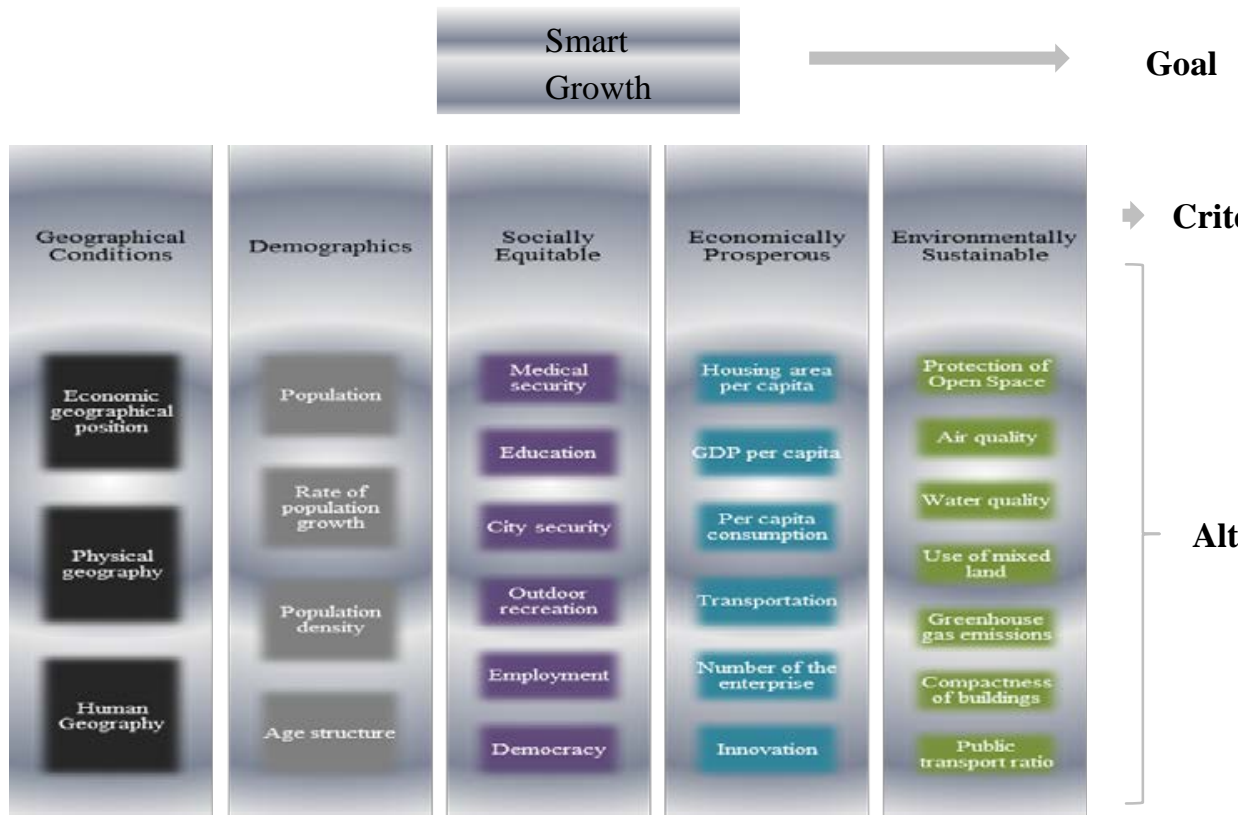

\section{Criteria}

Figure 1. The three-hierarchy structure

Also, the standard system should include transportation, health care, education, tourism and so on. Therefore, from Figure 1, we know clearly the establishment of urban smart growth standards.

\subsection{Obtain the index weight and test consistency}

Quantification of all the sub-factors is a huge and difficult task for many factors. Combining qualitative analysis with quantitative analysis, we use the APH method to assign appropriate weight to each index.

- Determine the judging matrix

According to the metrics system and the AHP model, we use the pairwise comparison method and one-nine method to construct judging matrix $A=\left(a_{i j}\right)$

$$
a_{i k} * a_{k j}=a_{i j}
$$

Where $a_{i j}$ is set according to the one-nine method by Stayy.

- Calculate the eigenvalues and eigenvectors

The greatest eigenvalue of matrix $\mathrm{A}$ is $\lambda_{\max }$, and the corresponding eigenvector is $u=\left(u_{1}, u_{2}, u_{3} \ldots u_{n}\right)^{T}$ Then we normalize the $\mathrm{u}$ by the expression:

$$
\omega_{i}=\frac{u_{i}}{\sum_{i=0}^{n} u_{j}}
$$

Do the consistency check

The indicator of consistency check formula:

$$
C I=\frac{\lambda-n}{n-1}
$$

Where $\mathrm{n}$ denotes the exponent number of matrix.

The expression of consistency ratio: 


$$
C R=\frac{C I}{R I}
$$

As we have confirmed the weighting coefficient of all the indicators in the evaluation system, now we quantify the importance of first level evaluation norms.

$\omega_{i}$ presents the weight of $K_{i}$. According to the above results can be obtained, the main factor expression:

We can easily compute that:

$$
\left\{\begin{array}{l}
\mathrm{F}=\sum_{i=1}^{6} \omega_{F i} F_{i} \\
S=\sum_{i=1}^{6} \omega_{s i} S_{i} \\
E=\sum_{i=1}^{7} \omega_{E i} E_{i} \\
\mathrm{G}=\sum_{i=1}^{3} \omega_{G i} G_{i} \\
P=\sum_{i=1}^{3} \omega_{P i} P_{i}
\end{array}\right.
$$

$$
\mathbf{C}=\boldsymbol{\omega}_{1} \sum_{i=1}^{6} \omega_{F i} F_{i}+\omega_{2} \sum_{i=1}^{6} \omega_{s i} S_{i}+\omega_{3} \sum_{i=1}^{7} \omega_{E i} E_{i}+\omega_{4} \sum_{i=1}^{3} \omega_{G i} G_{i}+\omega_{5} \sum_{i=1}^{3} \omega_{P i} P_{i}
$$

According to formula (6), the metric system to measure the success of smart growth of a city is achieved.

\subsection{Results}

Based on the data we have already collected, we solve the model and obtain the following results:

\section{- Judging matrix:}

Five aspects of criteria level is as follows:

$$
\left[\begin{array}{ccccc}
1 & 5 / 2 & 4 / 3 & 4 & 5 / 3 \\
2 / 5 & 1 & 1 / 2 & 8 / 5 & 2 / 3 \\
3 / 4 & 2 & 1 & 3 & 5 / 4 \\
1 / 4 & 5 / 8 & 1 / 3 & 1 & 1 / 2 \\
3 / 5 & 3 / 2 & 4 / 5 & 2 & 1
\end{array}\right]
$$

Also, judging matrix of alternatives level can easily get.

\section{- Weight vector of criteria level:}

$$
\omega=\left[\begin{array}{lllll}
0.334 & 0.1319 & 0.2538 & 0.0867 & 0.1935
\end{array}\right]^{T}
$$

For this level, $C I=0$ satisfying $\frac{C I}{R I}<0.1$.

\section{- Weight vector of alternatives level:}

All of these five vectors satisfy $\frac{C I}{R I}<0.1$.

Finally, it can be considered that the weight vector can be used as the weight of the factors of criteria level and alternatives level through the consistency test.

- Analysis of criteria level

From the above formulas can be obtained, the proportion of criteria level is as below: 


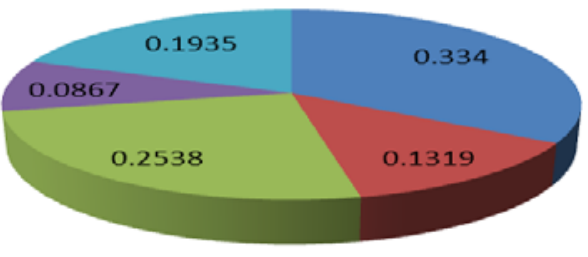

Economically Prosperous

= Socially Equitable

Environmentally

Sustainable

- Geographical Conditions

E Demographics

Figure 2. Weight vector of criteria level

In Figure 2, the economy plays the most important part in the development of a city. When the economy reaches a certain level, the population and the environment is particularly important, which is consistent with the development of cities around the world and indicates the feasibility of this model.

\section{- Analysis of alternatives level}

Because each factor has different influences on urban livability, the most practical method utilized is expert scoring system, taking the average of expert judgment as judging matrix.

\subsection{Classify our metrics}

We selected 12 cities of different sizes in different continents and collected the data of 26 indicators of alternatives level.

In order to avoid the effect of different dimensions and orders of magnitude on the results, we first normalize all the data. Then assume that the data samples for 12 countries are $X_{1}, X_{2} \ldots X_{12}$.

Matrix $\mathbf{X}$ is:

$$
\begin{gathered}
\mathbf{X}=\left[\begin{array}{lllll}
X_{1} & X_{2} & X_{3} & \ldots & X_{12}
\end{array}\right]^{T} \\
\mathbf{X}_{i}=\left[\begin{array}{lllll}
X_{i 1} & X_{i 2} & X_{i 3} & \ldots & X_{i 26}
\end{array}\right](i=1 \ldots 12)
\end{gathered}
$$

Formula (10) is normalized:

$$
\boldsymbol{X}^{*}=\left(x^{*}{ }_{i j}\right)_{12 \times 26}
$$

Meantime

$$
x_{i j}^{*}=\frac{x_{i j}-\overline{x_{j}}}{\sigma_{j}}(i=1,2 \ldots 12 ; j=1,2 \ldots 26)
$$

The normalized data are separately entered into the formula (6). We can get the ranking of each indicator of alternatives level

The dimension of data above is different, so the order of magnitude is also different. Because the calculation and query data are different, and the real data may have some errors, and other geographical factors are qualitative factors.

Scaling all city parameters into scores, we define the score of $M_{i}$ is $K_{i}$ :

$$
K_{i}=\frac{M_{i}}{M_{\max }} \times 100
$$

Then according to the formula (6):

$$
\mathrm{C}=\sum_{i=1}^{5} \omega_{i} K_{i}
$$

According to the weight of criteria level, the top of score is 85. From the table above, it can be obtained that only Fargo comprehensive score reached 80, we think that Fargo's smart growth level of A, then according to the above situation is divided into five grades in Table 1. 
Table 1. Ranking

\begin{tabular}{cc}
\hline Ranking & Score \\
\hline A & $80-100$ \\
B & $60-80$ \\
C & $40-60$ \\
D & $20-40$ \\
E & $0-20$
\end{tabular}

According to Table 4, we divide scores into five levels in descending order: A, B, C, D and E.

\section{References}

[1] World Urbanization $\quad$ Prospects. United 2014. https://esa.un.org/unpd/wup/Publications/Files/WUP2014-Highlights.pdf

[2] Wangzhenyuan. An AHP-based Approach to Evaluation Indicator System of China' s Smart City[J]. Science and Technology Management Research, 2014, (17): 166-167

[3] Jiangqiyuan. Analytic Hierarchy Process Model[C]. No.4, De Wai Avenue, Xicheng District, Beijing:Higher Education Press, 2006. 226-227

[4] Beijing:Higher Education Press, 2006. 226-227 